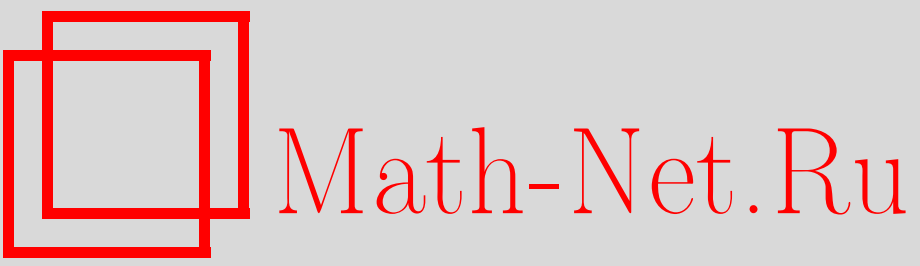

В. С. Герджиков, Б. Б. Байзаков, М. Салерно, Моделирование адиабатических $N$-солитонных взаимодействий с учетом возмущений, ТMФ, 2005, том 144, номер 2, 302-312

DOI: https://doi.org/10.4213/tmf1855

Использование Общероссийского математического портала Math-Net.Ru подразумевает, что вы прочитали и согласны с пользовательским соглашением

http: //www . mathnet.ru/rus/agreement

Параметры загрузки :

IP : 54.164 .48 .24

26 апреля 2023 г., 13:45:39 
ТЕОРЕТИЧЕСКАЯ

И МАТЕМАТИЧЕСКАЯ

ФИЗИКА

Том 144, № 2

август, 2005

(C) 2005 г. $\quad$ В. С. Герджиков ${ }^{*}$, Б. Б. Байзаков ${ }^{\dagger}$, М. Салерно $^{\dagger}$

\title{
МОДЕЛИРОВАНИЕ АДИАБАТИЧЕСКИХ $N$-СОЛИТОННЫХ ВЗАИМОДЕЙСТВИЙ С УЧЕТОМ ВОЗМУЩЕНИЙ
}

\begin{abstract}
С целью описания адиабатических взаимодействий $N$-солитонной цепочки для нелинейного уравнения Шредингера с возмущением исследуется вариант комплексной цепочки Тоды с возмушением. Возмущения в слабых квадратичном и периодическом потенциалах исследуются как аналитическими, так и численными методами. Комплексная цепочка Тоды с возмущением адекватно моделирует динамику $N$-солитонной цепочки для обоих типов потенциалов. В качестве приложения развитой теории рассматривается динамика цепочки солитонов волн материи, удерживаемой параболической ловушкой и оптической решеткой.
\end{abstract}

Ключевые слова: комплексная цепочка Тоды, адиабатическая динамика, солитонная цепочка, выталкивание солитона.

\section{1. ВВЕДЕНИЕ}

Взаимодействия $N$-солитонных цепочек для нелинейного уравнения Шредингера (НУШ) и его вариантов с возмушением

$$
i u_{t}+\frac{1}{2} u_{x x}+|u|^{2} u(x, t)=i \epsilon R[u]
$$

начало изучению которых положила новаторская работа [1], в настоящее время в значительной степени исследованы (см. работы [2]-[6] и цитируемую там литературу). Исследовался также и ряд других нелинейных эволюционных уравнений, в том числе некоторые высшие НУШ [6], система Абловица-Ладика [7], модифицированное НУШ [8]-[12] и другие.

\footnotetext{
*Institute for Nuclear Research and Nuclear Energy, 1784 Sofia, Bulgaria. E-mail: gerjikov@inrne.bas.bg

${ }^{\dagger}$ Department of Physics "E. R. Caianiello" and Istituto Nazionale di Fisica della Materia, University of Salerno, I-84081 Baronissi, Italy. E-mail: salerno@sa.infn.it
} 
Сосредоточимся далее на НУШ с возмушением (1). Под $N$-солитонной цепочкой мы понимаем решение НУШ (с возмушением), определенное начальным условием

$$
\begin{aligned}
u(x, t=0) & =\sum_{k=1}^{N} u_{k}^{1 \mathrm{~s}}(x, t=0), \quad u_{k}^{1 \mathrm{~s}}(x, t)=\frac{2 \nu_{k} e^{i \phi_{k}}}{\operatorname{ch} z_{k}}, \\
z_{k}(x, t) & =2 \nu_{k}\left(x-\xi_{k}(t)\right), \quad \xi_{k}(t)=2 \mu_{k} t+\xi_{k, 0}, \\
\phi_{k}(x, t) & =\frac{\mu_{k}}{\nu_{k}} z_{k}+\delta_{k}(t), \quad \delta_{k}(t)=W_{k} t+\delta_{k, 0} .
\end{aligned}
$$

Каждый солитон характеризуется четырьмя параметрами: амплитудой $\nu_{k}$, скоростью $\mu_{k}$, положением центра масс $\xi_{k}$ и фазой $\delta_{k}$. В адиабатическом приближении роль малого параметра $\varepsilon_{0} \ll 1$ играет перекрытие солитонов, которое экспоненциально уменьшается с расстоянием между солитонами. В таком случае параметры солитонов должны удовлетворять следуюшим условиям [1]:

$$
\left|\nu_{k}-\nu_{0}\right| \ll \nu_{0}, \quad\left|\mu_{k}-\mu_{0}\right| \ll \mu_{0}, \quad\left|\nu_{k}-\nu_{0}\right|\left|\xi_{k+1,0}-\xi_{k, 0}\right| \gg 1,
$$

где $\nu_{0}=(1 / N) \sum_{k=1}^{N} \nu_{k}$ и $\mu_{0}=(1 / N) \sum_{k=1}^{N} \mu_{k}-$ средние амплитуда и скорость, соответственно. На самом деле мы имеем два разных масштаба:

$$
\left|\nu_{k}-\nu_{0}\right| \simeq \varepsilon_{0}^{1 / 2}, \quad\left|\mu_{k}-\mu_{0}\right| \simeq \varepsilon_{0}^{1 / 2}, \quad\left|\xi_{k+1,0}-\xi_{k, 0}\right| \simeq \varepsilon_{0}^{-1} .
$$

Можно ожидать, что приближение применимо только для таких времен $t$, для которых набор $4 N$ параметров солитонной цепочки удовлетворяет условиям (5).

Уравнение (1) находит ряд применений в нелинейной оптике, а в случае $R[u] \equiv 0$ интегрируемо методом обратной задачи рассеяния [13], [14]. Динамика $N$-солитонной цепочки в адиабатическом приближении моделируется посредством комплексного обобщения цепочки Тоды [20]:

$$
\frac{d^{2} Q_{j}}{d t^{2}}=16 \nu_{0}^{2}\left(e^{Q_{j+1}-Q_{j}}-e^{Q_{j}-Q_{j-1}}\right), \quad j=1, \ldots, N .
$$

Комплекснозначные величины $Q_{k}$ выражаются через параметры солитонов:

$$
Q_{k}(t)=2 i \lambda_{0} \xi_{k}(t)+2 k \ln \left(2 \nu_{0}\right)+i\left(k \pi-\delta_{k}(t)-\delta_{0}\right),
$$

где $\delta_{0}=(1 / N) \sum_{k=1}^{N} \delta_{k}$ и $\lambda_{0}=\mu_{0}+i \nu_{0}$. Кроме того, предполагается, что концы цепочки свободны, т.е. $e^{-Q_{0}} \equiv e^{Q_{N+1}} \equiv 0$.

Заметим, что $N$-солитонная цепочка не является $N$-солитонным решением: спектральные данные соответствующего оператора Лакса $L$ не являются тривиальными и в непрерывной части спектра $L$. Поэтому аналитические результаты теории солитонов не могут быть применены. Кроме того, мы хотим рассмотреть солитоны, двигающиеся с равными скоростями, а также эффекты возможных неинтегрируемых возмушений $R[u]$. 
Представленная статья расширяет результаты работ [2]-[5], [12], [15]. В настоящее время в связи с реализацией в разреженных атомарных газах конденсации Бозе-Эйнштейна стало важно исследовать НУШ с дополнительным потенциальным членом вида $i R[u]=V(x) u(x, t)$ (см. [16], [17]). Мы продолжаем начатый в работе [6] анализ соответствуюшей модели комплексной цепочки Тоды (КЦТ) с возмущением для квадратичных и периодических потенциалов $V(x)$. Наши результаты подтверждают наличие у периодических потенциалов стабилизационных свойств, которые были обнаружены в работах [18], [19] в другой физической постановке.

\section{2. ЗНАЧЕНИЕ МОДЕЛИ КЦТ}

Тот факт [20], что КЦТ, подобно (вешественной) цепочке Тоды (ВЦТ), является вполне интегрируемой гамильтоновой системой, позволяет аналитически исследовать асимптотическое поведение $N$-солитонных цепочек. Однако КЦТ в отличие от ВЦТ обладает большим многообразием динамических режимов [21], а именно:

- асимптотически свободное движение в случае, если $v_{j} \neq v_{k}$ при $j \neq k$; это единственный динамический режим, возможный для ВЦТ;

- $N$-солитонное связанное состояние в случае, если $v_{1}=\cdots=v_{N}$, а $\zeta_{k} \neq \zeta_{j}$ при $k \neq j$

- различные промежуточные (смешанные) режимы; например, если $v_{1}=v_{2}>\cdots$ $\cdots>v_{N}$, а $\zeta_{k} \neq \zeta_{j}$ при $k \neq j$, то будем иметь связанное состояние первых двух солитонов, в то время как остальные будут асимптотически свободными;

- сингулярные и вырожденные режимы в случае, если два или более собственных значений оператора $L$ равны, например, $\zeta_{1}=\zeta_{2}, \ldots$ и $\zeta_{j} \neq \zeta_{k}$ при $2<j \neq k$.

Здесь через $\zeta_{k}=v_{k}+i w_{k}$ обозначены собственные значения матрицы Лакса $L$ в представлении Лакса $L_{\tau}=[M, L]$ для КЩТ, где

$$
\begin{gathered}
L=\sum_{k=1}^{N} b_{k} E_{k k}+\sum_{k=1}^{N-1} a_{k}\left(E_{k, k+1}+E_{k+1, k}\right), \\
b_{k} \equiv-\frac{1}{2} \frac{d Q_{k}}{d \tau}=\frac{1}{2}\left(\mu_{k}+i \nu_{k}\right), \quad a_{k}=\frac{1}{2} \exp \frac{Q_{k+1}-Q_{k}}{2}
\end{gathered}
$$

и $\left(E_{k p}\right)_{i j}=\delta_{k i} \delta_{p j}$. Собственные значения $\zeta_{k}$ оператора $L$ не зависят от времени и являются комплекснозначными наряду с первыми компонентами нормированных собственных векторов $L$ :

$$
L \vec{z}^{(k)}=\zeta_{k} \vec{z}^{(k)}, \quad\left(\vec{z}^{(k)}, \vec{z}^{(m)}\right)=\delta_{k m} .
$$

Множество $\left\{\zeta_{k}=v_{k}+i w_{k}, \eta_{k}=\sigma_{k}+i \theta_{k}\right\}$ может рассматриваться как множество переменных действие-угол для КЦТ.

Используя модель КЦТ, можно определить асимптотический режим $N$-солитонной цепочки. По заданным начальным параметрам $\mu_{k}(0), \nu_{k}(0), \xi_{k}(0), \delta_{k}(0) N$-солитонной цепочки можно вычислить матричные элементы $b_{k}$ и $a_{k}$ оператора $L$ при $t=0$. Затем, решая характеристическое уравнение для $\left.L\right|_{t=0}$, можно вычислить собственные значения $\zeta_{k}$ и таким образом определить асимптотический режим $N$-солитонной цепочки 
[2], [5]. Другая возможность состоит в том, чтобы наложить на $\zeta_{k}$ специальное ограничение, например, потребовать, чтобы все $\zeta_{k}$ были чисто мнимыми, т.е. $v_{k}=0$ для всех $k$. Это даст множество алгебраических условий для $\left.L\right|_{t=0}$ и начальных параметров солитонов $\mu_{k}(0), \nu_{k}(0), \xi_{k}(0), \delta_{k}(0)$, которые описывают область в пространстве солитонных параметров, отвечающую за $N$-солитонные связанные состояния.

\section{3. НУШ И КЦТ С ВОЗМУЩЕНИЯМИ. КВАДРАТИЧНЫЙ И ПЕРИОДИЧЕСКИЙ ПОТЕНЦИАЛЫ}

Рассмотрим несколько специальных способов выбора возмущений $R^{(p)}[u], \quad p=$ $1,2, \ldots$, в уравнении (1). Динамика параметров солитонов в адиабатическом приближении может быть определена с помошью следуюшей системы (см. [1] для $N=2$ и [2], [5] для $N>2$ ):

$$
\begin{gathered}
\frac{d \lambda_{k}}{d t}=-4 \nu_{0}\left(e^{Q_{k+1}-Q_{k}}-e^{Q_{k}-Q_{k-1}}\right)+M_{k}^{(p)}+i N_{k}^{(p)}, \\
\frac{d \xi_{k}}{d t}=2 \mu_{k}+\Xi_{k}^{(p)}, \quad \frac{d \delta_{k}}{d t}=2\left(\mu_{k}^{2}+\nu_{k}^{2}\right)+X_{k}^{(p)},
\end{gathered}
$$

где $\lambda_{k}=\mu_{k}+i \nu_{k}$ и $X_{k}^{(p)}=2 \mu_{k} \Xi_{k}^{(p)}+D_{k}^{(p)}$. Правые части уравнений (10), (11) определяются величиной $R_{k}^{(p)}[u]$ :

$$
\begin{aligned}
N_{k}^{(p)} & =\frac{1}{2} \int_{-\infty}^{\infty} \frac{d z_{k}}{\operatorname{ch} z_{k}} \operatorname{Re}\left(R_{k}^{(p)}[u] e^{-i \phi_{k}}\right), \\
M_{k}^{(p)} & =\frac{1}{2} \int_{-\infty}^{\infty} \frac{d z_{k} \operatorname{sh} z_{k}}{\operatorname{ch}^{2} z_{k}} \operatorname{Im}\left(R_{k}^{(p)}[u] e^{-i \phi_{k}}\right), \\
\Xi_{k}^{(p)} & =\frac{1}{4 \nu_{k}^{2}} \int_{-\infty}^{\infty} \frac{d z_{k} z_{k}}{\operatorname{ch} z_{k}} \operatorname{Re}\left(R_{k}^{(p)}[u] e^{-i \phi_{k}}\right), \\
D_{k}^{(p)} & =\frac{1}{2 \nu_{k}} \int_{-\infty}^{\infty} \frac{d z_{k}\left(1-z_{k} \operatorname{th} z_{k}\right)}{\operatorname{ch} z_{k}} \operatorname{Im}\left(R_{k}^{(p)}[u] e^{-i \phi_{k}}\right) .
\end{aligned}
$$

Подставляя (10), (11) в (7), получаем

$$
\begin{gathered}
\frac{d Q_{k}}{d t}=-4 \nu_{0} \lambda_{k}+\frac{2 k}{\nu_{0}} \mathcal{N}_{0}^{(p)}+2 i \xi_{k}\left(\mathcal{M}_{0}^{(p)}+i \mathcal{N}_{0}^{(p)}\right)+i\left(2 \lambda_{0} \Xi_{k}^{(p)}-X_{k}^{(p)}-\mathcal{X}_{0}^{(p)}\right), \\
\mathcal{N}_{0}^{(p)}=\frac{1}{N} \sum_{j=1}^{N} N_{j}^{(p)}, \quad \mathcal{M}_{0}^{(p)}=\frac{1}{N} \sum_{j=1}^{N} M_{j}^{(p)}, \quad \mathcal{X}_{0}^{(p)}=\frac{1}{N} \sum_{j=1}^{N} X_{j}^{(p)} .
\end{gathered}
$$

При выводе формул (16) мы оставили члены порядка $\Delta \nu_{k} \simeq \mathcal{O}\left(\sqrt{\epsilon_{0}}\right)$ и пренебрегли членами порядка $\mathcal{O}\left(\epsilon_{0}\right)$. В результате возмушений величины $\nu_{0}$ и $\mu_{0}$ могут стать не зависяшими от времени. Действительно, из уравнения (10) получаем

$$
\frac{d \mu_{0}}{d t}=\mathcal{M}_{0}^{(p)}, \quad \frac{d \nu_{0}}{d t}=\mathcal{N}_{0}^{(p)}
$$

4 Теоретическая и математическая физика, т. 144, № 2, 2005 г. 
Мальй параметр $\epsilon_{0}$ может быть связан с начальным расстоянием $r_{0}=\left|\xi_{2}-\xi_{1}\right|_{t=0}$ меж ду двумя солитонами. Предполагая $\nu_{1,2} \simeq \nu_{0}$, находим

$$
\epsilon_{0}=\int_{-\infty}^{\infty} d x\left|u_{1}^{1 \mathrm{~s}}(x, 0) u_{2}^{1 \mathrm{~s}}(x, 0)\right| \simeq 8 \nu_{0} r_{0} e^{-2 \nu_{0} r_{0}} .
$$

Соотношение (18), в частности, означает, что $\epsilon_{0} \simeq 0.01$ при $r_{0} \simeq 8$ и $\nu_{0}=1 / 2$.

Будем предполагать, что в начальный момент солитоны упорядочены таким образом, что $\xi_{k+1}-\xi_{k} \simeq r_{0}$. Можно проверить [3], [12], что $N_{k}^{(p)} \simeq M_{k}^{(p)} \simeq \exp \left(-2 \nu_{0}|k-p| r_{0}\right)$. Поэтому члены, описываюшие взаимодействие между $k$-м и $(k \pm 1)$-м солитонами, будут порядка $e^{-2 \nu_{0} r_{0}}$, взаимодействие между $k$-м и $(k \pm 2)$-м солитонами -порядка $e^{-4 \nu_{0} r_{0}} \ll$ $e^{-2 \nu_{0} r_{0}}$

Члены $\Xi_{k}^{(0)}, X_{k}^{(0)}$ по порядку величины равны $r_{0}^{a} e^{-2 \nu_{0} r_{0}}$, где $a=0$ или 1 . Однако этими членами можно пренебречь по сравнению с $\tilde{\mu}_{k}$ и $\tilde{\nu}_{k}$ :

$$
\tilde{\mu}_{k}=\mu_{k}-\mu_{0} \simeq \sqrt{\epsilon_{0}}, \quad \tilde{\nu}_{k}=\nu_{k}-\nu_{0} \simeq \sqrt{\epsilon_{0}} .
$$

Поправки к $N_{k}^{(p)}, \ldots$, получаемые из линейных по $u$ членов, зависят только от параметров $k$-го солитона, т.е. они “локальны” по $k$. Представленные в $i R^{(p)}[u]$ нелинейные по $u$ члены дают, кроме того, "нелокальные" по $k$ члены в $N_{k}^{(p)}, \ldots$

Рассмотрим теперь возмушения вида $i R[u]=V(x) u(x, t)$. Первый способ выбора $V(x)$ - квадратичный:

$$
V^{(1)}(x)=V_{2} x^{2}+V_{1} x+V_{0} .
$$

Опустив детали, имеем в результате [6]

$$
\begin{aligned}
& N_{k}^{(1)}=0, \quad M_{k}^{(1)}=-V_{2} \xi_{k}-\frac{V_{1}}{2}, \\
& \Xi_{k}^{(1)}=0, \quad D_{k}^{(1)}=V_{2}\left(\frac{\pi^{2}}{48 \nu_{k}^{2}}-\xi_{k}^{2}\right)-V_{1} \xi_{k}-V_{0}
\end{aligned}
$$

и $X_{k}^{(1)}=D_{k}^{(1)}$. Другой важный способ выбора - периодический потенциал

$$
V^{(2)}(x)=A \cos \left(\Omega x+\Omega_{0}\right),
$$

где $A, \Omega$ и $\Omega_{0}$ - подходяшим образом выбранные константы. НУШ с аналогичными потенциалами естественным образом возникает при изучении конденсата Бозе-Эйнштейна (см. [16]). Для двух взаимодействуюших солитонов соответствуюшая система Карпмана-Соловьева была получена в работе [19]. В случае $N>2$ мы получаем КЦТ с возмушением (КЦТВ), причем интегралы для величин $N_{k}, M_{k}, \Xi_{k}$ и $D_{k}$ равны [6] соответственно

$$
\begin{aligned}
& N_{k}^{(2)}=0, \quad M_{k}^{(2)}=\frac{\pi A \Omega^{2}}{8 \nu_{k}} \frac{1}{\operatorname{sh} Z_{k}} \sin \left(\Omega \xi_{k}+\Omega_{0}\right), \\
& \Xi_{k}^{(2)}=0, \quad D_{k}^{(2)}=-\frac{\pi^{2} A \Omega^{2}}{16 \nu_{k}^{2}} \frac{\operatorname{ch} Z_{k}}{\operatorname{sh}^{2} Z_{k}} \cos \left(\Omega \xi_{k}+\Omega_{0}\right),
\end{aligned}
$$

где $Z_{k}=\pi \Omega /\left(4 \nu_{k}\right)$. Эти результаты позволяют получить соответствующие модели КЦТВ. 


\section{4. АНАЛИЗ КЦТВ И СРАВНЕНИЕ С ЧИСЛЕННЫМ МОДЕЛИРОВАНИЕМ}

Динамика отдельного солитона в цепочке определяется совместным действием внешнего потенциала и влиянием соседних солитонов. Сила взаимодействия между соседними солитонами может быть как силой отталкивания, так и силой притяжения в зависимости от фазовых соотношений между ними. В частности, если амплитуды солитонов равны между собой, а начальная разность фаз между соседними солитонами равна $\pi$ (этот случай рассматривается ниже), то такие солитоны отталкивают друг друга, что в отсутствие внешнего поля вызывает растяжение солитонной цепочки [2], [3].

Внешний потенциал противодействует этому растяжению, стараясь удержать солитоны в минимумах потенциала. Именно совместное действие этих двух факторов - взаимодействия солитонов и действия внешнего потенциала - порождает богатую динамику $N$-солитонной цепочки.

Чтобы удостовериться в адекватности модели КЦТВ для описания динамики $N$-солитонной цепочки во внешнем потенциале, мы осуществили сравнение предсказаний на основе соответствуюшей системы КЦТВ с результатами прямого численного моделирования для базового НУШ (1). Ниже представлены результаты, относящиеся к солитонам волн материи в удерживающей параболической ловушке и в периодическом потенциале, моделируюшем оптическую решетку.

4.1. Квадратичный потенциал. Рассмотрим динамику $N$-солитонной цепочки, удерживаемой квадратичным потенциалом $V(x)=V_{2} x^{2}+V_{1} x+V_{0}$. Это приложение представляет практический интерес, поскольку имеет прямое отношение к недавним экспериментам с цепочками солитонов в конденсате Бозе-Эйнштейна [22]. Теоретический анализ цепочек в конденсате Бозе-Эйнштейна в периодических ловушках, включающий метод решетки Тоды, был представлен в работе [17]. Соответствуюшая модель КШТВ в терминах солитонных параметров имеет вид

$$
\begin{aligned}
& \frac{d \mu_{k}}{d t}= 16 \nu_{0}^{3}\left(e^{-2 \nu_{0}\left(\xi_{k+1}-\xi_{k}\right)} \cos \left(2 \mu_{0}\left(\xi_{k+1}-\xi_{k}\right)+\delta_{k}-\delta_{k+1}\right)-\right. \\
&\left.-e^{-2 \nu_{0}\left(\xi_{k}-\xi_{k-1}\right)} \cos \left(2 \mu_{0}\left(\xi_{k}-\xi_{k-1}\right)+\delta_{k-1}-\delta_{k}\right)\right)-V_{2} \xi_{k}-\frac{V_{1}}{2} \\
& \frac{d \nu_{k}}{d t}= 16 \nu_{0}^{3}\left(e^{-2 \nu_{0}\left(\xi_{k+1}-\xi_{k}\right)} \sin \left(2 \mu_{0}\left(\xi_{k+1}-\xi_{k}\right)+\delta_{k}-\delta_{k+1}\right)-\right. \\
&\left.-e^{-2 \nu_{0}\left(\xi_{k}-\xi_{k-1}\right)} \sin \left(2 \mu_{0}\left(\xi_{k}-\xi_{k-1}\right)+\delta_{k-1}-\delta_{k}\right)\right) \\
& \frac{d \xi_{k}}{d t}= 2 \mu_{k} \\
& \frac{d \delta_{k}}{d t}=2\left(\mu_{k}^{2}+\nu_{k}^{2}\right)+V_{2}\left(\frac{\pi^{2}}{48 \nu_{k}^{2}}-\xi_{k}^{2}\right)-V_{1} \xi_{k}-V_{0}
\end{aligned}
$$

где $\mu_{k}, \nu_{k}, \xi_{k}$ и $\delta_{k}$ суть скорость, амплитуда, положение центра масс и фаза $k$-го солитона, и $\mu_{0}$ и $\nu_{0}-$ средние скорость и амплитуда цепочки. 
Разумно предположить, что $V_{2} \simeq \mathcal{O}\left(\epsilon_{0} / N\right)$; это предположение обеспечивает возможность получения $N$-солитонной цепочки "внутри" потенциала. Это также означает, что экспоненциальные члены и поправки $M_{k}^{(1)}$ имеют один порядок величины. Тогда уравнение $(26)$ дает $d \nu_{0} / d t=0$, в то время как из уравнений $(25),(27)$ получаем

$$
\frac{d \mu_{0}}{d t}=-V_{2} \xi_{0}-\frac{V_{1}}{2}, \quad \frac{d \xi_{0}}{d t}=2 \mu_{0}
$$

где $\mu_{0}$ - средняя скорость, а $\xi_{0}=(1 / N) \sum_{j=1}^{N} \xi_{j}$-положение центра масс $N$-солитонной цепочки. Система уравнений (29) при $V_{2}>0$ имеет простое решение

$$
\mu_{0}(t)=\mu_{00} \cos (\Phi(t)), \quad \xi_{0}(t)=\sqrt{\frac{2}{V_{2}}} \mu_{00} \sin (\Phi(t))-\frac{V_{1}}{2 V_{2}}
$$

где $\Phi(t)=\sqrt{2 V_{2}} t+\Phi_{0}$, а $\mu_{00}$ и $\Phi_{0}-$ постоянные интегрирования. Из уравнений $(29)$ находим, что $d \nu_{0} / d t=0$. Поэтому итоговое действие такого квадратичного потенциала вызывает медленное периодическое движение цепочки как целого.

Если положение центра масс $N$-солитонной цепочки совпадает с минимумом квадратичного потенциала, то солитоны совершают движение, выражаюшееся в периодическом сжатии и растяжении цепочки, как показано на рис. 1.

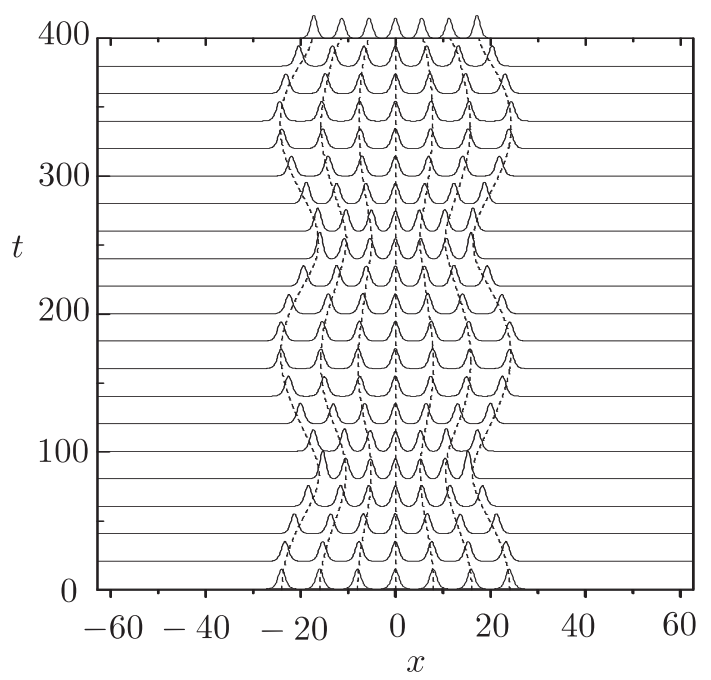

Рис.1. 7-солитонная цепочка в параболическом захватывающем потенциале $V(x)=$ $0.0001 x^{2}$ совершает периодические колебания (сжимается и растягивается) вокруг центра захвата. Сплошные линии соответствуют результатам прямого численного моделирования НУШ (1), штриховые линии - результату решения КЦТВ системы (25)-(28) для центра масс $\xi_{i}$. Начальные разность фаз и расстояние между соседними солитонами равны соответственно $\pi$ и 8 . Солитоны имеют одинаковые амплитуды $2 \nu=1$. 
Динамика $N$-солитонной цепочки, расположенной несимметрично относительно центра параболической ловушки, является более сложной. Центр масс цепочки совершает гармонические колебания вокруг минимума потенциала, и одновременно отдельные солитоны участвуют в движении, результатом которого является сжатие и растяжение цепочки. Картина движения представлена на рис. 2. Из уравнений (30) следует, что период колебаний центра масс равен $T=2 \pi / \sqrt{2 V_{2}}$; для параметров, отвечающих рис. 2 , имеем $T=314.2$, что хорошо согласуется с результатом численного моделирования.

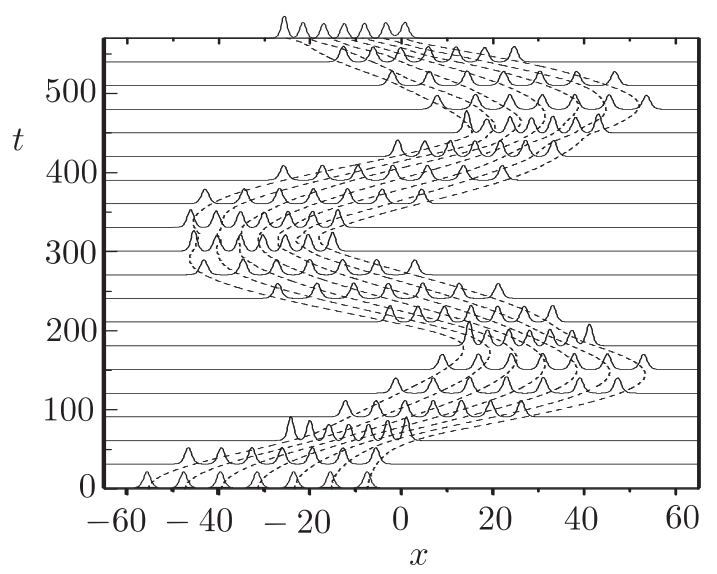

Рис. 2. Динамика 7-солитонной цепочки, расположенной несимметрично относительно минимума потенциала ловушки $V(x)=0.0002 x^{2}$. Сплошные линии соответствуют результатам прямого численного моделирования НУШ (1), штриховые линии - результату решения КЦТВ системы (25)-(28) для центра масс $\xi_{i}$. Параметры солитонов такие же, как на рис. 1. Начальное смещение цепочки солитонов равно $-10 \pi$.

Прямое моделирование НУШ (1) показывет, что достаточно сильная параболическая ловушка может вызывать слияние отдельных солитонов во время сжатия цепочки с восстановлением исходной конфигурации в то время, когда цепочка растянута. Такое поведение напоминает экспериментально наблюдаемое явление "исчезновения солитонов" [22]. Впрочем, эта ситуация выходит за рамки применимости КцТВ-подхода.

4.2. Периодический потенциал. Другим внешним потенциалом, в котором $N$-солитонная цепочка демонстрирует интересную динамику, является периодический потенциал вида $V(x)=A \cos \left(\Omega x+\Omega_{0}\right)$. Этот случай также может иметь прямое отношение к цепочкам солитонов волн материи, удерживаемым оптическими решетками. Система КЦТВ в терминах солитонных параметров имеет вид

$$
\begin{aligned}
\frac{d \mu_{k}}{d t}= & 16 \nu_{0}^{3}\left(e^{-2 \nu_{0}\left(\xi_{k+1}-\xi_{k}\right)} \cos \left(2 \mu_{0}\left(\xi_{k+1}-\xi_{k}\right)+\delta_{k}-\delta_{k+1}\right)-\right. \\
& \left.-e^{-2 \nu_{0}\left(\xi_{k}-\xi_{k-1}\right)} \cos \left(2 \mu_{0}\left(\xi_{k}-\xi_{k-1}\right)+\delta_{k-1}-\delta_{k}\right)\right)+M_{k}^{(2)}\left(\nu_{k}\right),
\end{aligned}
$$




$$
\begin{aligned}
\frac{d \nu_{k}}{d t}= & 16 \nu_{0}^{3}\left(e^{-2 \nu_{0}\left(\xi_{k+1}-\xi_{k}\right)} \sin \left(2 \mu_{0}\left(\xi_{k+1}-\xi_{k}\right)+\delta_{k}-\delta_{k+1}\right)-\right. \\
& \left.-e^{-2 \nu_{0}\left(\xi_{k}-\xi_{k-1}\right)} \sin \left(2 \mu_{0}\left(\xi_{k}-\xi_{k-1}\right)+\delta_{k-1}-\delta_{k}\right)\right), \\
\frac{d \xi_{k}}{d t}= & 2 \mu_{k}, \\
\frac{d \delta_{k}}{d t}= & 2\left(\mu_{k}^{2}+\nu_{k}^{2}\right)+D_{k}^{(2)}\left(\nu_{k}\right),
\end{aligned}
$$

где величины $M_{k}^{(2)}\left(\nu_{k}\right)$ и $D_{k}^{(2)}\left(\nu_{k}\right)$ даются формулами (23) и (24).

На каждый солитон цепочки действует удерживающая сила периодического потенциала и отталкиваюшая сила соседних солитонов. Поэтому положения равновесия солитонов не совпадают с минимумами периодического потенциала. Солитоны, расположенные первоначально в минимумах периодического потенциала (рис. 3), совершают колебания малой амплитуды вокруг этих минимумов при условии, что сила потенциала достаточна для удержания солитонов. В противоположность этому слабый периодический потенциал не в состоянии удержать солитоны, и силы отталкивания между соседними солитонами (с разностью фаз $\pi$ ) приводят к неограниченному растяжению цепочки.

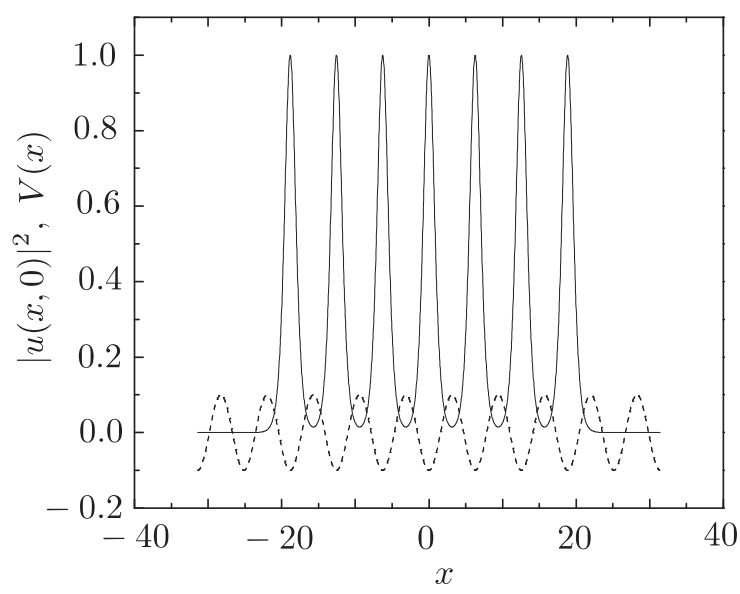

Рис. 3. Солитоны (сплошная линия) удерживаются около минимумов периодического потенциала $V(x)=A \cos (x)$ (штриховая линия), совершая колебания малой амплитуды, в случае, если сила потенциала достаточно велика $A=-0.1$.

В переходной области, когда сила удержания периодического потенциала сравнима по величине с силой отталкивания соседних солитонов, наблюдается интересная динамика, например, выталкивание граничных солитонов из цепочки, как показано на рис. 4 .

Это явление, демонстрируюшее сложность внутренней динамики цепочки, может быть объяснено следуюшим образом. Каждый солитон совершает нелинейные колебания внутри отдельной потенциальной ямы под действием силы отталкивания со стороны соседних солитонов. Когда амплитуда колебаний отдельных солитонов возрастает и два солитона подходят близко друг к другу, сильньй отталкиваюший импульс может 


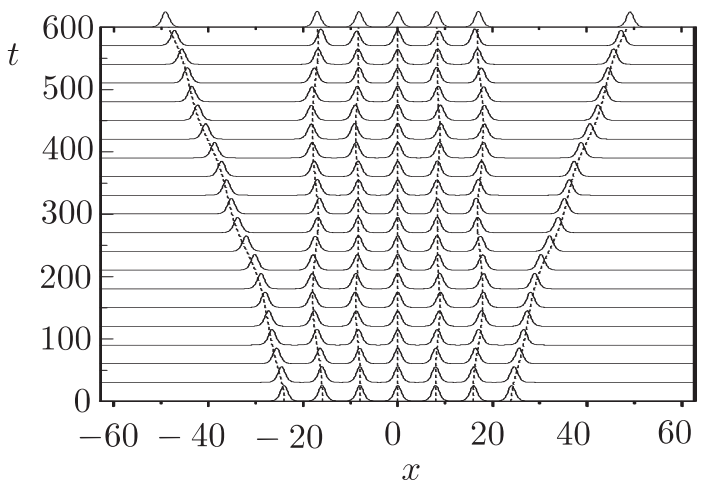

Рис. 4. Выталкивание солитонов из цепочки, полученное в результате прямого численного моделирования НУШ (1) (сплошные линии) и рассчитанное с помощью КЦТВ системы (31)-(34) для центра масс $\xi_{i}$ (штриховые линии). Амплитуды солитонов одинаковы и равны $2 \nu=1$, разности фаз и расстояния между соседними солитонами соответственно $\pi$ и 8 . Периодический потенциал выбран в виде $V(x)=-0.001 \cos (\pi x / 4)$.

стать причиной того, что солитон, преодолев периодический потенциальный барьер, покинет цепочку. На рис. 4 это происходит с граничными солитонами (остальные солитоны остаются связанными в течение долгого времени эволюции). Стоит подчеркнуть, что это явление хорошо описывается в рамках модели КЦТВ, как ясно видно из рис. 4 .

\section{5. ЗАКЛЮЧЕНИЕ}

Мы исследовали динамику $N$-солитонной цепочки, удерживаемой во внешних полях (в квадратичном и периодическом потенциалах). Как аналитическое рассмотрение в рамках модели КЦТВ, так и численный анализ посредством прямого моделирования основного НУШ показывают, что КЦТВ адекватно описывает $N$-солитонные взаимодействия во внешних потенциалах. Мы кратко остановились на том, какое отношение имеет данная работа к исследованию солитонов волн материи, удерживаемых магнитными ловушками и оптическими решетками.

Благодарности. В. С. Герджиков благодарит М. Бойти, Ф. Пемпинелли и Б. Принари за предоставленную возможность участвовать в конференции "Нелинейная физика. Теория и эксперимент. ІІІ" и университет г. Лечче за гостеприимство; И. Узунова за полезные обсуждения, а также National Science Foundation of Bulgaria за финансовую поддержку, контракт № F-1410. Б. Б. Байзаков благодарит Department of Physics at the University of Salerno, Италия, за исследовательский грант. М. Салерно благодарен за частичную финансовую поддержку, оказанную MIUR в рамках межуниверситетского проекта PRIN-2003, и Istituto Nazionale di Fisica Nucleare, sezione di Salerno.

\section{Список литературы}

[1] V.I. Karpman, V. V. Solov'ev. Physica D. 1981. V. 3. P. 487.

[2] V.S. Gerdjikov, D. J. Kaup, I. M. Uzunov, E. G. Evstatiev. Phys. Rev. Lett. 1996. V. 77. P. 3943. 
[3] V.S. Gerdjikov, I. M. Uzunov, E. G. Evstatiev, G.L. Diankov. Phys. Rev. E. 1997. V. 55. № 5. P. 6039.

[4] J. M. Arnold. J. Opt. Soc. Am. A. 1998. V. 15. P. 1450; Phys. Rev. E. 1999. V. 60. P. 979.

[5] V.S. Gerdjikov, E. G. Evstatiev, D.J. Kaup, G.L. Diankov, I. M. Uzunov. Phys. Lett. A. 1998. V. 241. P. 323.

[6] V.S. Gerdjikov. On Modelling Adiabatic $N$-soliton Interactions. Effects of perturbations. In: Nonlinear Waves: Classical and Quantum Aspects. Proc. NATO Adv. Research Workshop. (Estoril, Portugal, 13-17 July 2003). NATO Sci. Ser. II. Math. Phys. Chem. V. 153. Eds. F. Kh. Abdullaev, V. V. Konotop. Dordrecht: Kluwer, 2004. P. 15.

[7] E. V. Doktorov, N. P. Matsuka, V. M. Rothos. Phys. Rev. E. 2004. V. 69. P. 056607.

[8] Y. Kodama, A. Hasegawa. IEEE J. Quant. Electr. 1987. V. QE-23. P. 510.

[9] В.С. Герджиков, М.И. Иванов, П. П. Кулиш. ТМФ. 1980. Т. 44. С. 342 ; В. С. Герджиков, М.И. Иванов. Bulg. J. Phys. 1983. V. 10. № 1. Р. 13; № 2. P. 130. (In Russian).

[10] V.S. Shchesnovich, E. V. Doktorov. Physica D. 1999. V. 129. P. 115; J. Math. Phys. 1995. V. 36. P. 7009.

[11] V.S. Gerdjikov, E. V. Doktorov, J. Yang. Phys. Rev. E. 2001. V. 64. P. 056617.

[12] V.S. Gerdjikov, I. M. Uzunov. Physica D. 2001. V. 152-153. P. 355.

[13] В. Е. Захаров, С. В. Манаков, С. П. Новиков, Л. П. Питаевский. Теория солитонов. Метод обратной задачи. М.: Наука, 1980.

[14] Л. А. Тахтаджян, Л. Д. Фаддеев. Гамильтонов подход в теории солитонов. М.: Наука, 1986.

[15] V.S. Gerdjikov. Complex Toda chain - an integrable universal model for adiabatic $N$-soliton interactions. In: Nonlinear Physics: Theory and Experiment. II. Proc. Int. Workshop (Lecce, Italy, 2002). Eds. M. Ablowitz, M. Boiti, F. Pempinelli, B. Prinari. River Edge, NJ: World Scientific, 2003. P. 186.

[16] J. C. Bronski, L. D. Carr, B. Deconinck, J. N. Kutz. Phys. Rev. E. 2001. V. 63. P. 036612; Phys. Rev. Lett. 2001. V. 86. P. 1402; J. C. Bronski, L. D. Carr, R. Carretero-Gonzalez, B. Deconinck, J. N. Kutz, K. Promislow. Phys. Rev. E. 2001. V. 64. P. 056615; F. Kh. Abdullaev, B. B. Baizakov, S. A. Darmanyan, V. V. Konotop, M. Salerno. Phys. Rev. A. 2001. V. 64. P. 043606.

[17] R. Carretero-Gonzalez, K. Promislow. Phys. Rev. A. 2002. V. 66. P. 033610.

[18] S. Wabnitz. Electron. Lett. 1993. V. 29. P. 1711.

[19] I. M. Uzunov, M. Gölles, F. Lederer. J. Opt. Soc. Am. B. 1995. V. 12. № 6. P. 1164.

[20] C. В. Манаков. ЖЭЭФ. 1974. Т. 67. С. 543; H. Flaschka. Phys. Rev. B. 1974. V. 9. P. 1924.

[21] J. Moser. Dynamical systems, finitely many mass points on the line under the influence of an exponential potential - an integrable system. In: Dynamical Systems, Theory and Applications. Lecture Notes in Phys. V.38. Ed. J. Moser. Berlin: Springer, 1975. P. 467; V. S. Gerdjikov, E. G. Evstatiev, R. I. Ivanov. J. Phys. A. 1998. V. 31. P. 8221.

[22] K. E. Strecker, G. B. Partridge, A. G. Truscott, R. G. Hulet. Nature. 2002. V. 417. P. 150. 